\title{
Ultrasound Elastography Based on Multiscale Estimations of Regularized Displacement Fields
}

\author{
Claire Pellot-Barakat*, Member, IEEE, Frédérique Frouin, Member, IEEE, Michael F. Insana, Member, IEEE, and \\ Alain Herment, Member, IEEE
}

\begin{abstract}
Elasticity imaging is based on the measurements of local tissue deformation. The approach to ultrasound elasticity imaging presented in this paper relies on the estimation of dense displacement fields by a coarse-to-fine minimization of an energy function that combines constraints of conservation of echo amplitude and displacement field continuity. The multiscale optimization scheme presents several characteristics aimed at improving and accelerating the convergence of the minimization process. This includes the nonregularized initialization at the coarsest resolution and the use of adaptive configuration spaces. Parameters of the energy model and optimization were adjusted using data obtained from a tissue-like phantom material. Elasticity images from normal in vivo breast tissue were subsequently obtained with these parameters. Introducing a smoothness constraint into motion field estimation helped solve ambiguities due to incoherent motion, leading to elastograms less degraded by decorrelation noise than the ones obtained from correlation-based techniques.
\end{abstract}

Index Terms-Motion estimation, multiscale optimization, optical flow, regularization, ultrasound elastography.

\section{INTRODUCTION}

$\mathbf{E}$ LASTICITY imaging describes the compressibility of biological tissues [1]. Changes in tissue stiffness correlate with pathological phenomena and can be indicators of major diseases, such as cancer [2]-[5] or cardiovascular disease [6], [7]. Various elasticity imaging techniques have been developed over the past decade. Reviews are found in papers by Gao [1], Parker [8], Wilson [9], and Ophir [10].

Quantitative assessment of elasticity can be provided by measuring elastic parameters such as strain [11], which is inversely proportional to the elastic modulus of tissue [12]. Strain images or elastograms are usually computed based on measurements of local deformations induced by compressive forces and captured using conventional imaging modalities, mainly ultrasound and magnetic resonance imaging. Most approaches to ul-

Manuscript received May 5, 2003; revised August 26, 2003. This work was supported in part by the National Institutes of Health (NIH) under Grant ROI CA 82497. The Associate Editor responsible for coordinating the review of this paper and recommending its publication was M. Sonka. Asterisk indicates corresponding author.

${ }^{*}$ C. Pellot-Barakat is with the Department of Biomedical Engineering, University of California, Davis, CA 95616 USA, on leave from the French National Institute of Health (INSERM) U494 Paris, France (e-mail: cjbarakat@ ucdavis.edu).

F. Frouin is with INSERM, U494 Paris, France (e-mail: frouin@ imed.jussieu.fr).

M. F. Insana is with the Department of Biomedical Engineering, University of California, Davis, CA 95616 USA (e-mail: mfinsana@ucdavis.edu).

A. Herment is with INSERM, U494 Paris, France (e-mail: herment@ imed.jussieu.fr).

Digital Object Identifier 10.1109/TMI.2003.822825 trasonic elasticity imaging consist of computing strain estimates as the spatial gradient of local tissue displacements. Techniques have also been proposed that estimate strain directly without involving estimation of displacement, either within the framework of inverse problem solutions [13]-[16] or using the power spectra of signals [17]. The approach described in this paper falls into the first category of methods. It addresses the problem of constructing high quality strain images by precise estimation of local movements of tissues under deformation.

Most ultrasonic techniques estimate local displacements of scatterers based on correlation analysis of echoes [2], [3], [18]-[20]. Cross-correlation is a very accurate and effective estimator of distance (or similarity) between echo fields and is capable of tracking small variations even when very low strains are involved. This estimator is robust to noise. However, strain imaging is subjected to ultrasonic speckle decorrelation induced by out-of-plane motion, nonuniform motion of subresolution scatterers, nonuniformity of the ultrasound field and nonrigid tissue deformation [21]. Speckle decorrelation results in loss of echo signal coherence and leads to displacement estimation errors [12]. Attempts have been made to reduce decorrelation noise and increase the range of accurate strain measurements using adaptive methods that compute local scaling factors [19], [22]-[24]. Companding methods consisting of joint operations of compression and expansion of echo fields prior to cross-correlation have also been proposed in order to compensate for scaling and shifting of echoes in the scan plane and help restore signal coherence [25], [26]. The multiscale companding method originally proposed by [25] and modified by introducing a semi-constrained correlation search based on physical priors of tissue motion continuity [27] provides elastograms of in vivo breast and vascular tissues with high spatial resolution and contrast. However, elastograms remain degraded by decorrelation noise, especially when large and out of plane motion is present. In fact, incoherent motion and variations in the signal from scatterers at high compression lead to ambiguities in the determination of the motion vectors [26].

Since data alone can be insufficient to solve ambiguities due to loss of echo coherence, we propose an approach that integrates a priori knowledge into the motion estimation process. Regularization provides the theoretical framework necessary to integrate this additional information. Due to the complexity and spatial heterogeneity of motion, a global parametric regularization model is inappropriate. Optical flow seems a better model as it permits estimation of local deformations. Methods for computing optical flow were first proposed by Horn and Shunk [28] and applied to medical imaging by Mailloux et al. [29]. Optical flow is based on the implicit assumptions that signal amplitudes are constant between sequentially recorded frames and that local texture con- 
tains sufficient unambiguous information [30]. However, the hypothesis that signal intensities remain constant along the motion trajectories is not fully justified, especially in the case of ultrasound elasticity imaging where compression of scatterers leads to changes in their echo response [31]. Consequently, estimating the optical flow from local information is ill-posed. To regularize this problem, approaches based on a Markovian model of optical flow have been proposed [32], [33]. The method we developed is based on a regularization of the optical flow using an approach similar to that described by Perez and Heitz [32]. The algorithm estimates the optical flow by minimizing a global function that reflects the typical constraint of conservation of the echo amplitude while imposing a smoothness constraint on the displacement field. The search of the minimum difference energy configuration is performed using a deterministic multiscale descent optimization algorithm [34].

An integral part of the formulation of regularization problems is the choice of the smoothness parameter that controls the tradeoff between noise reduction and resolution and contrast degradation. The regularizing factor as well as other key optimization parameters was adjusted empirically using phantom and breast tissue data obtained with different sets of parameters. For that purpose, elastograms obtained with different regularizations were qualitatively compared with elastograms obtained with a multiscale cross-correlation technique used as the reference method. Performances in terms of noise and contrast were also measured and compared.

Section II focus on the description of the specific regularized model and associated multiscale optimization strategy proposed to reconstruct the displacement field associated to ultrasonic radio frequency (RF) data. The adjustment of the regularization parameters and the subsequent elasticity imaging results from phantom and breast data are discussed in Section III.

\section{METHODS}

\section{A. Elasticity Estimation}

Elasticity imaging can be performed using static or dynamic stimuli [9]. In this paper, only static elastography is considered, where external compressing forces are applied at the skin surface. An ultrasonic transducer flush mounted onto a small plexiglass compression plate facilitates application of a uniform stress distribution. Elasticity measurements involve tracking the locations of scatterers in tissue from a resting state to a compressed state. In order to increase the sensitivity and precision of displacement estimates, RF data are preferred over B-mode envelope data. $\mathrm{RF}$ echoes are acquired along A-lines in the rest and compressed states. A-lines are divided into small segments, and the postdeformation displacement $d$ is estimated for each segment. We estimate strain along the beam axis where the wave modulation increases the sensitivity of echo signals to motion. Strain estimates $\varepsilon$ for each segment of initial length $L$ are estimated using

$$
\varepsilon=\Delta d / L \text {. }
$$

Managing three-dimensional tissue motion is a major factor affecting experimental design and algorithm development [25]. Assuming all motion of the biological media can be confined to a plane, for example, by adjusting boundary conditions, then precise estimates of axial displacements may be obtained by analyzing echoes in the image plane. Nevertheless, differences in sampling interval for different directions with respect to the beam axis complicate algorithm development and limit precision.

The axial sampling interval $(\Delta z)$ used here is $53.5 \mu \mathrm{m}$ while the lateral sampling interval $(\Delta y)$ is $120 \mu \mathrm{m}$. In order to increase the precision of displacement estimates, RF data are interpolated by a factor of 4 in the axial direction. Elastograms are then obtained by calculating the axial strain as the first-order finite difference of the estimated displacement field in the $z$ direction. Since the differentiation operator is very sensitive to noise, displacement estimates are averaged along $z$ (kernel size: $1.7 \mathrm{~mm}$, overlapping: $214 \mu \mathrm{m}$ ) prior to differentiation to limit degradation of the signal to noise ratio.

\section{B. Regularized Modeling of the Optical Flow}

We seek to estimate the dense displacement field $d=$ $\{d(s), s \in S\}$ corresponding to a pair of frames from the RF image sequence $f=\left\{f_{\sigma}(s), s \in S\right\}$ defined over the rectangular grid $S$ of size $Z \times Y$. The movement is represented with respect to the first frame of the echo sequence, corresponding to the resting state $(\sigma=0)$. A pixel site $s$ with coordinates $(z, y)$ in the reference frame will move after compression by a factor $\sigma$ to the location $s+d(s)$ with $d(s)=\left(d_{a}(s), d_{l}(s)\right) d_{a}$, and $d_{l}$ representing the axial and lateral components of $d$. Displacement vector solutions have values in a discrete configuration set that depends on experimental parameters such as sampling specifics, boundary conditions, and the applied force stimulus. The set $\Lambda$ of possible displacements (search window) is defined as

$$
\Lambda=\left\{-D_{a}, \ldots, D_{a}\right\} \times\left\{-D_{l}, \ldots, D_{l}\right\}
$$

where $D_{a}$ is the maximum authorized value of displacement in the axial direction $(z)$ and $D_{l}$ is the maximum authorized value of displacement in the lateral direction $(y)$. The set of all possible configurations is $\Omega=\Lambda^{Z \times Y}$.

To estimate displacement vectors, spatial variations of the echo amplitude $f$ as function of the applied compressive force are analyzed in the framework of regularized optical flow (ROF). For that purpose, we define an energy cost function that is constrained by echo amplitude conservation and neighborhood smoothness of displacements. It is then minimized in order to find the most probable value of the displacement field $\hat{d}$. Specifically

$$
\hat{d}=\arg \min _{d \in \Omega}\left(E_{1}(d)+\alpha E_{2}(d)\right)
$$

where $\alpha$ is a positive regularization factor.

The first term assumes echo amplitude is conserved. It corresponds to the shifted inter-echo image difference. A simple sumabsolute difference (SAD) measure is used, which has demonstrated accuracy comparable to the usual correlation measure and requires less computation [11], [35]. It is written as

$$
E_{1}(d)=\sum_{s \in S}\left|f_{0}(s)-f_{\sigma}(s+d(s))\right|
$$

The second term of (3) regularizes the solutions by minimizing local variations of the vector field. It is defined as

$$
E_{2}(d)=\sum_{s \in S} \sum_{s^{\prime} \in C_{s}}\left\|d(s)-d\left(s^{\prime}\right)\right\|^{2}
$$

where $C_{s}$ represents an 8-sample neighborhood about site $s$. 


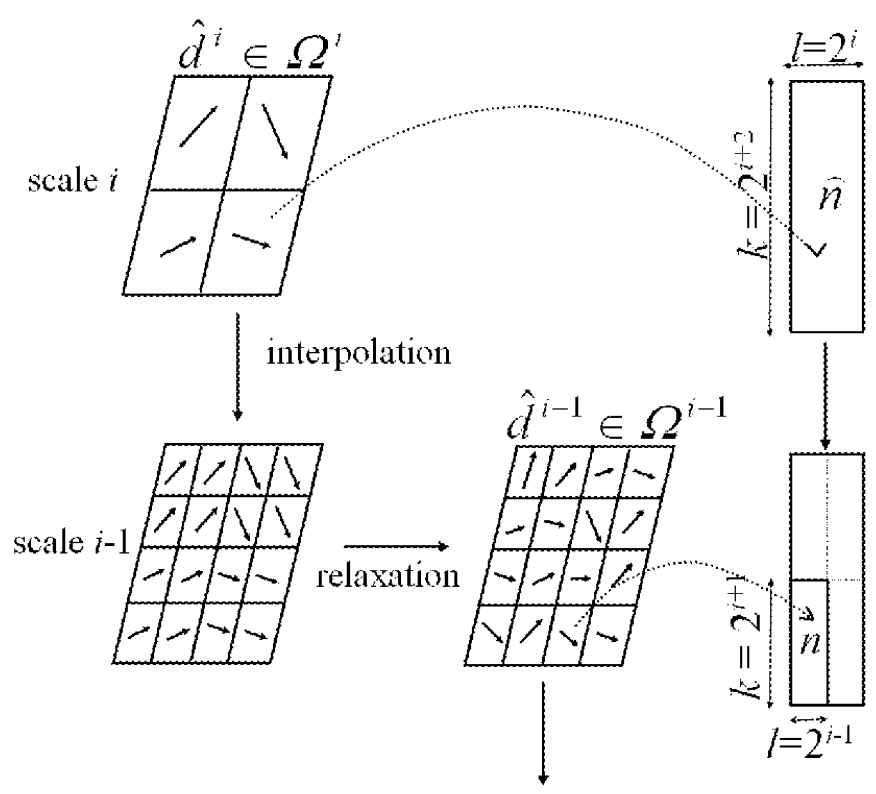

Fig. 1. (a) Multiscale optimization: The minimization of the energy at level $i$ leads to the estimation of the displacement field $\hat{d}^{i}$ which is interpolated to the resolution of the next scale $i-1$. A new estimate $\hat{d}^{i+1}$ of the displacement field is obtained, which is again interpolated. The same operations are repeated until $i=0$.

\section{Multiscale Optimization Adapted to Nonisotropic Data}

The search for the minimum energy is a classical optimization problem. The energy function we seek to minimize is nonconvex and presents several local minima. Stochastic algorithms such as Simulated Annealing converge in theory toward a global minima of the energy function [36]. However, stochastic relaxation algorithms necessitate a large number of iterations and are very slow, especially when the field to be estimated is large (high value of $Z \times Y$ ). They are not well suited to our application, where minimizing computation time is an important consideration. Deterministic algorithms such as the iterative conditional modes (ICM) algorithm [37] converge much faster but can be trapped in local minima of the energy function. In order to avoid selecting local minima, optimization can be initialized with configurations that are close to the global minimum [34]. Multiscale or pyramidal approaches offer this possibility by searching for the solution within finer and finer spaces of configurations. In contrast to multiresolution approaches that observe data and measure displacements at increasing spatial resolutions, the multiscale approach uses the finest spatial resolution for all observations but varies the scale of the configuration space $\Omega$. The optimization strategy we have implemented is an adaptation of multiscale ICM algorithms to the specific problem of anisotropically sampled ultrasonic RF data.

Optimization consists of satisfying (3) while searching for solutions of the displacement field $d$ within $M=I+1$ successive spaces of configurations $\Omega^{i}(i=I, I-1, \ldots, 0)$, included in the original space $\Omega$, and obtained by grouping blocks of sites Fig. 1. Multiscale algorithms presented in the literature only consider the specific case of isotropically sampled data, and use spaces of configurations built from square blocks. Since investigated RF data have a significantly higher axial than lateral sampling, anisotropic blocks have been introduced in this paper. The ratio of axial and lateral block dimensions is normally set at 4 to account for the axial interpolation. The displacement field is estimated over a rectangular grid $S$ of dimension $Z \times Y$ that is fit within the original field of view, where $Z$ and $Y$ are multiples of $4 \times 2^{I}$ and $2^{I}$. For configuration scale $i$, the grid is partitioned into $N_{i}$ rectangular blocks $B_{n}^{i}$ of size $k \times l$ Fig. 1 . This design leads to $k=2^{i+2}, l=2^{i}$, and $N_{i}=Z \times Y / 2^{2 i+2}$. At each scale $i$ is associated a configuration $d^{i}$ defined over a grid $S^{i}$. Displacement vectors within the same block $B_{n}^{i}$ are identical

$$
\forall n \in\left\{1, \ldots, N_{i}\right\}, \quad \forall s \in B_{n}^{i}, \quad d(s)=d^{i}(n) .
$$

At each scale, we estimate the suite of configurations

$$
\hat{d}^{i}=\arg \min _{d^{i} \in \Omega^{i}}\left(E_{1}^{i}\left(d^{i}\right)+\alpha_{i} E_{2}^{i}\left(d^{i}\right)\right) .
$$

Note that at the finest configuration scale $(i=0)$, blocks are of size $4 \times 1$. This leads to estimation of the displacement field according to the original resolution (before axial interpolation) but with an accuracy of the axial component of $\Delta z / 4$.

During ICM optimization, sites from the grid are visited sequentially at each iteration. To prevent the formation of artifacts due to a privileged scanning direction, alternative scans starting at the top left, bottom right, top right, and bottom left of the grid are performed. To ensure that a stable minimum energy is reached at a given spatial scale $i$, iterations should ideally stop when no more sites are modified. However, each time the scale increases a factor of 2, the number of sites visited increases a factor 4. At coarse scales, convergence is achieved within a few iterations, but at fine scales, many iterations are necessary to achieve the same stable state. To homogenize the optimization, iterations are stopped when the number of modified sites during an iteration becomes smaller than a threshold $\eta_{i}$ function of the scale $i$. This threshold is chosen so that ICM optimization is stopped at the coarsest scales when none of the sites is modified during a full scanning, and at finest scales when the number of modified sites is lower than one per cent of the number of visited sites

$$
\begin{cases}\eta_{i}=0, & \text { for } i>3 \\ \eta_{i}=N_{i} / 100, & \text { for } i \leq 3\end{cases}
$$

\section{Nonregularized Initialization}

In conventional multiscale approaches, the same cost function is used at each level so that the energy models at different scales are completely determined by the original model corresponding to the finest scale. Stated mathematically

$$
\forall i \in\{0, \ldots, I\},\left\{\alpha_{i}=\alpha, E_{1}^{i}\left(d^{i}\right)=E_{1}(d), E_{2}^{i}\left(d^{i}\right)=E_{2}(d)\right\} .
$$

where $d$ is related to $d^{i}$ according to (6).

However, regularization at the highest level (coarsest scale) can lead to an initial solution that is too smooth and, thus, traps solutions in local minima, as will be illustrated in Section III-3. We have considered that the displacement vector field is already regularized because all the sites of a block are estimated by a constant vector; hence, we chose to cancel the regularization cost for $i=I$, i.e., $\alpha_{I}=0$. It is not necessary to iterate in that case, since only the conservation term intervenes in the energy model. Optimization at the coarsest scale, thus, becomes a simple minimum SAD search with a finite number of values where the energy only depends on the echo data and is not affected by the estimates. This nonregularized model, which is 
only used at the top level, can be, in a sense, considered as a general initialization. All the other resolution levels use the same energy model $\left(\forall i \in\{0, \ldots, I-1\}, \alpha_{i}=\alpha\right)$ so that the energy model remains coherent along the different scales.

For a given scale $i$, the amplitude conservation term $E_{1}^{i}\left(d^{i}\right)$ is defined as

$$
\begin{aligned}
E_{1}^{i}\left(d^{i}\right) & =\sum_{n=1}^{N_{i}} \sum_{s \in B_{n}^{i}}\left|f_{0}(s)-f_{\sigma}\left(s+d^{i}(n)\right)\right| \\
& =\sum_{s \in S}\left|f_{0}(s)-f_{\sigma}(s+d(s))\right|
\end{aligned}
$$

which, using the relationship from (6), is identical to the definition of $E_{1}$ from (4).

The regularization energy is also independent of the scale. At a given scale $i$, the regularization term can be written as

$$
E_{2}^{i}\left(d^{i}\right)=\sum_{n=1}^{N_{i}} \sum_{s \in B_{n}^{i}} \sum_{s^{\prime} \in C_{s}}\left\|d^{i}(n)-d\left(s^{\prime}\right)\right\|^{2}
$$

which is identical to the definition of $E_{2}$ in (5).

However, since displacement vectors are constant within a block, the local displacement variation cost will be null for neighboring pixels that are within the same block. The computation time of $E_{2}^{i}$ can be considerably reduced by excluding same block neighbors. A simplified expression of (11) can be obtained as

$$
E_{2}^{i}\left(d^{i}\right)=\sum_{n=1}^{N_{i}} \sum_{n^{\prime} \in C_{n}^{i}} \lambda_{c}^{i}\left\|d^{i}(n)-d^{i}\left(n^{\prime}\right)\right\|^{2}
$$

where $\lambda_{c}^{i}$ is determined by the number of adjacent pixels in neighboring blocks $\left\{B_{n}^{i}, B_{n^{\prime}}^{i}\right\}$, and $C_{n}^{i}$ refers to the neighborhood system associated with $B_{n}^{i}$. We decompose $C_{n}^{i}$ into three subneighboring systems $C_{n_{h}}^{i}, C_{n_{v}}^{i}$, and $C_{n_{g}}^{i}$, respectively referring to sets of horizontal, vertical, and diagonal neighbors of $d^{i}$. Equation (12) can be written as

$$
\begin{aligned}
E_{2}^{i}\left(d^{i}\right)=\sum_{n=1}^{N_{i}}[ & \sum_{n^{\prime} \in C_{n_{h}}^{i}} \lambda_{\mathrm{h}}^{i}\left\|d^{i}(n)-d^{i}\left(n^{\prime}\right)\right\|^{2} \\
& +\sum_{n^{\prime} \in C_{n_{v}}^{i}} \lambda_{\mathrm{v}}^{i}\left\|d^{i}(n)-d^{i}\left(n^{\prime}\right)\right\|^{2} \\
& \left.+\sum_{n^{\prime} \in C_{n_{g}}^{i}} \lambda_{\mathrm{g}}^{i}\left\|d^{i}(n)-d^{i}\left(n^{\prime}\right)\right\|^{2}\right]
\end{aligned}
$$

where $\lambda_{h}^{i}, \lambda_{v}^{i}$, and $\lambda_{g}^{i}$ are normalization factors corresponding to the number of pixels shared by a pair of neighboring blocks in the horizontal, vertical, and diagonal neighboring systems respectively. Since rectangular blocks are considered, horizontal, and vertical neighbors have different weights. In the horizontal neighboring system, $B_{n}^{i}$ possesses $k$ neighboring pixels with $B_{n^{\prime}}^{i}$ as shown in Fig. 2(a). Each of the two corner pixels on the side of $B_{n}^{i}$ adjacent to $B_{n^{\prime}}^{i}$ has 2 neighbors with $B_{n^{\prime}}^{i}$. The remaining $(k-2)$ noncorner pixels each has 3 neighbors with $B_{n^{\prime}}^{i}$. This leads to a total of $(2 \times 2+(k-2) \times 3)$ possible pairs of neighbors for $C_{n_{h}}^{i}$. In the vertical system, a total of $(2 \times 2+(l-2) \times 3)$ pairs of neighbors are possible [Fig. 2(b)]. Finally in the diagonal system, only one pair of pixels are neighbors [Fig. 2(c)].
Since $k=2^{i+2}$ and $l=2^{i}$, the normalization coefficients for a given scale $i$ are defined as

$$
\left\{\lambda_{h}^{i}=\left(3 \times 2^{i+2}-2\right), \lambda_{v}^{i}=\left(3 \times 2^{i}-2\right), \lambda_{g}^{i}=1\right\} .
$$

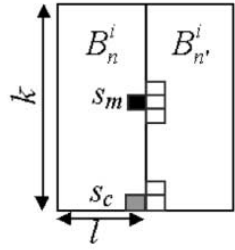

$$
\begin{aligned}
& \text { non-corner } \\
& \text { pixel of } B_{n}^{i} \\
& \square \text { of } B_{n}^{i} \\
& \left\{B_{n}^{i}, B_{n^{\prime}}^{i}\right\} \in C_{i}^{h}
\end{aligned}
$$

(a)

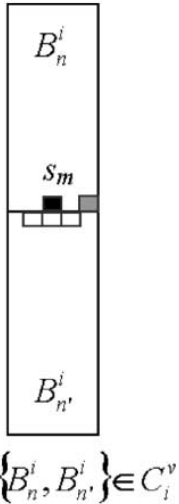

(b)

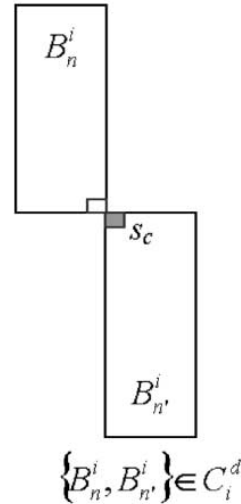

(c)
Fig. 2. Neighboring systems for blocks $B_{n}^{i}$. (a) In the horizontal neighboring system $B_{n}^{i}$ possesses $k$ pixels adjacent to $B_{n^{\prime}}^{i}$. Each of the 2 corner pixels $\left(s_{c}\right)$ of the side of $B_{n}^{i}$ adjacent to $B_{n^{\prime}}^{i}$ has 2 neighbors with $B_{n^{\prime}}^{i}$. The remaining $(k-2)$ noncorner pixels $\left(s_{m}\right)$ each has 3 neighbors with $B_{n^{\prime}}^{n}$. This leads to a total of $(2 \times 2+(k-2) \times 3)$ possible pairs of neighbors. (b) In the vertical system (middle), a total of $(2 \times 2+(l-2) \times 3)$ pairs of neighbors are possible. (c) In the diagonal system (right), only 1 pair of pixels are neighbors.

\section{E. Introduction of Adaptive Configuration Spaces}

Another characteristic of classical multiscale approaches is that the same search window is used at each scale [32]

$$
\forall i \in\{0, \ldots, I\}, \quad \Lambda^{i}=\Lambda
$$

leading to the configuration spaces

$$
\Omega^{i}=\Lambda^{N_{i}} .
$$

In our approach, search windows $\Lambda_{n}^{i}$ depending on the scale as well as on the studied block itself are defined. This allows more flexibility in the search of the minimum of the energy function as well as reduction of the computation time. Configuration spaces are defined as a function of the scale $i$ as

$$
\Omega^{i}=\prod_{n \in\left\{1, \ldots, N_{i}\right\}} \Lambda_{n}^{i} .
$$

1) Search Window at Scale I: When tissue displacements result from internal forces within organs (such as pulsation), no privileged direction of deformation is observed. However, when external forces are applied using the transducer, the displacements of scatterers at the end of the echo will be significantly larger than those at the beginning, since all measurements are relative to the transducer. For example, a uniform object compressed under ideal boundary conditions will exhibit a displacement that varies directly proportional to depth along $z$. Assuming a compression factor $\sigma$ is applied at the surface of a homogeneous object lying on an immovable support boundary, the linear displacement as a function of the axial depth is given by $d_{\operatorname{lin}}(z)=\sigma \times z$. Consequently, the search window at the bottom of the echo field is chosen larger than that at the top, and it is shifted to account for the axial scaling (Fig. 3). At the 
(a)

Table as reference
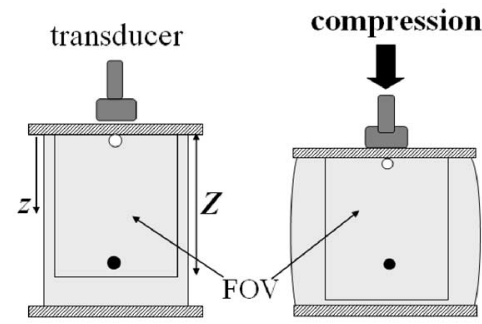

(b)

Transducer as reference
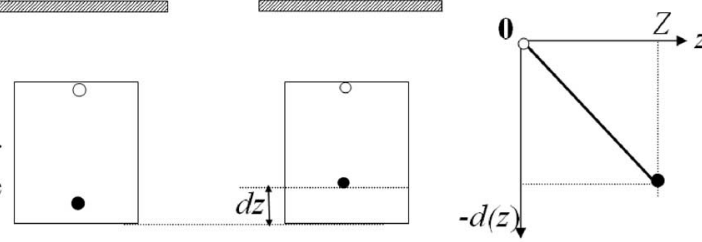

Fig. 3. (a) Axial compression of a homogeneous object with the ultrasonic transducer. (b) Precompression and postcompression acquired images (c) Resulting displacement (in the reference of the transducer) as a function of the axial direction $z$. The scatterer at the bottom of the object $(\bullet)$ is subject to the largest displacement.

coarsest scale $I$, the search window $\Lambda_{n}^{I}$ depends on $z_{n}$ defined as the $z$ value corresponding to the middle of $B_{n}^{I}$

$$
\begin{aligned}
\Lambda_{n}^{I}=\left\{d_{\operatorname{lin}}\left(z_{n}\right)-D_{a}, \ldots, d_{\operatorname{lin}}\left(z_{n}\right)\right. & \left.+D_{a}\right\} \\
& \times\left\{-D_{l}, \ldots, d_{l}\right\} .
\end{aligned}
$$

2) Search Window at Scale $i<I$ : Once a coarse estimation of the displacements has been made at scale $I$, search windows can be reduced and centered about the coarse estimates. The axial search extent at lower levels is chosen twice smaller than that of the top level. Search window centers are determined locally by the estimates found at the previous level.

Another modification consists of adaptively reducing the local search window in homogeneous areas. For that, a matrix $m^{i}(i \in\{0, \ldots, I-1\})$ of zeros is defined over the grid $S$. For sites $s$ belonging to $B_{n}^{i}$, the value $m^{i}(s)$ is incremented each time the estimate $\hat{d}^{i}(n)$ is modified. The counting of modified sites starts at scale $I-1$, since at level $I$, only one iteration is needed. If $\widehat{n}$ represents the block at $i+1$ from which $n$ originates, then search windows at the beginning of each scale optimization process, are adaptively defined for each block $n$ as

$$
\begin{aligned}
& \forall i< I, \\
& \Lambda_{n}^{i}=\left\{\hat{d}_{a}^{i+1}(\widehat{n})-D_{a} / 2, \ldots, \hat{d}_{a}^{i+1}(\widehat{n})+D_{a} / 2\right\} \\
& \times\left\{\hat{d}_{l}^{i+1}(\widehat{n})-D_{l}, \ldots, \hat{d}_{l}^{i+1}(\widehat{n})+D_{l}\right\} \\
& \text { if } m^{i+1}(\widehat{n})>0 \\
& \Lambda_{n}^{i}=\left\{\hat{d}_{a}^{i+1}(\widehat{n})-1, \hat{d}_{a}^{i+1}(\widehat{n}), \hat{d}_{a}^{i+1}(\widehat{n})+1\right\} \\
& \times\left\{\hat{d}_{l}^{i+1}(\widehat{n})-1, \hat{d}_{l}^{i+1}(\widehat{n}), \hat{d}_{l}^{i+1}(\widehat{n})+1\right\} \\
& \text { if } m^{i+1}(\widehat{n})=0 .
\end{aligned}
$$

This reduced search is based on the observation that neighboring sites belonging to elastically homogeneous regions will converge toward the optimal solution at the highest levels. If the displacement estimate of a block has not been modified during the relaxation process at scale $i$, this probably means that this block belongs to a homogeneous region and its estimate has reached a stable state. The adaptive window allows only a limited shift of \pm 1 pixel of the estimate in the four sub-blocks originating from a block that was found stable during the optimiza- tion at scale $i+1$. This limited search window was shown to be of sufficient size to account for the precision increase from one scale to the next.

\section{RESULTS}

\section{A. Motion Data Investigated}

Parameters of the ROF algorithm were adjusted using phantom data. A soft tissue-mimicking gelatin phantom was constructed with a stiff cylindrical inclusion designed to represent the conditions of a breast lesion. The inclusion was about six times stiffer than the phantom background. It also contains a soft and thin (1.5-mm diameter) fluid-filled channel. During strain imaging, the top surface of the phantom was compressed downward with the transducer face while the bottom phantom surface was fixed by a support. All exterior phantom surfaces were free to slip across any contacting boundary and fluid was free to flow in the channel. The method was then tested using data of normal breast tissue from volunteers acquired in vivo. Freehand scanning techniques were applied [3], [4] where out-of-plane motion cannot be controlled and large decorrelation errors are common for large deformations. To limit the effects of incoherent motion, small pressures $(<1 \%$ compression) were applied to the surface of the breast. Small compressions reduce decorrelation noise at the expense of strain image contrast.

Phantom construction and data acquisition are described by [38]. Radio-frequency data were reconstructed from IQ data acquired using a digital interface to a Siemens Sonoline Elegra system (Issaquah, WA) with a 7.5-MHz linear array. RF signals were digitized at $36 \mathrm{Msamples/s}$, downsampled by 2.5 for electronic transfer, then upsampled by 4 before processing to increase the precision of displacement estimates. Our patient scanning procedure is described elsewhere [27].

In the following section, characteristics of algorithmic optimization are illustrated using phantom data. Reconstructed elastograms from in vivo breast data are then shown. Although there is no reference standard to validate the strain results directly, the simplicity of the phantom structure and our prior experience [26] with finite-element modeling of similar phantoms allows us to anticipate the result with confidence. Elastograms obtained with our ROF algorithm are compared with those obtained using the well-tested multiresolution companding/correlation (MRCC) algorithm originally developed by Chatuverdi et al., [25], [26] and modified in a recent work [27] to allow adaptive search windows in order to reduce the computation time. The modified Chatuverdi algorithm is referred to as the MRCCA method in the following paragraphs.

\section{B. Parameter Adjustments}

The ROF method requires adjustment of three key parameters: regularization coefficient, number of scales and search extend. These parameters were empirically selected using phantom data. Criteria used to select adequate parameters were based on visual image appearance and convergence time as well as on comparison with the MRCC method. Measures of contrast-to noise ratio (CNR) were also performed to evaluate the performance of the algorithms in a more quantitative way. Although CNR is not a complete measure of target detectability, it provides a metric for comparing techniques 


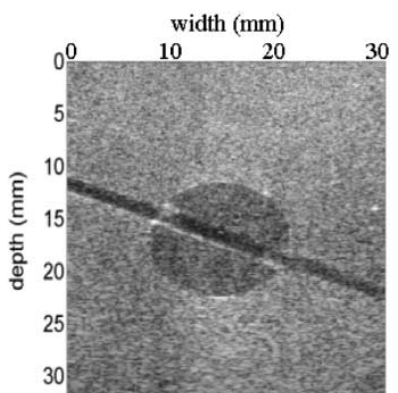

(a)

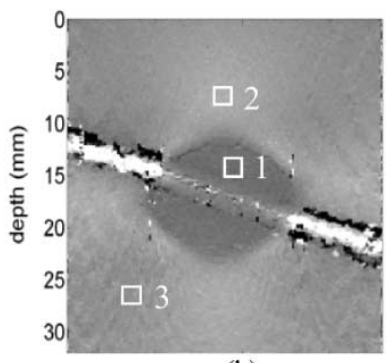

(b)

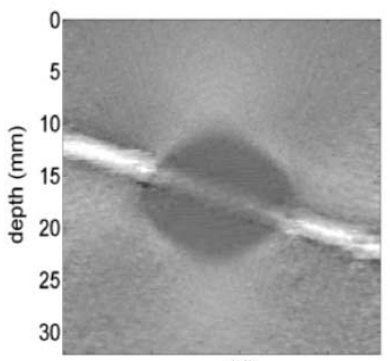

(d)

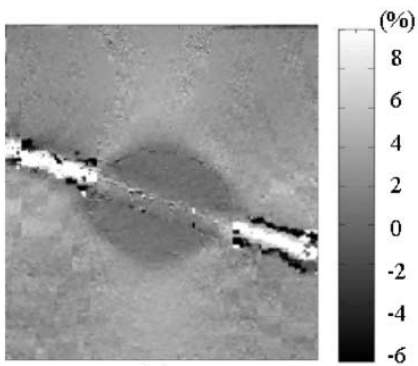

(c)

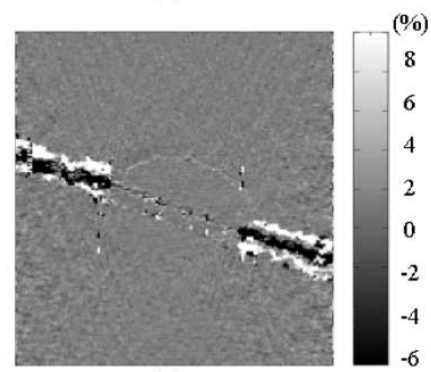

(e)
Fig. 4. (a) B-scan of a gelatin phantom containing a rigid cylindrical inclusion and a soft channel. (b) Corresponding elastogram obtained using an MRCC-based method (MRCC). The inclusion appears dark (low strain), while the channel appears bright (high strain) with decorrelation noise due to the flow. The channel is not as deformed inside the inclusion as it is outside. (c) Corresponding MRCCA elastogram. Some line artifacts are present. (d) Corresponding ROF elastogram. Decorrelation noise is eliminated. (e) Difference image between the MRCC and ROF elastograms.

based on large target contrast and relatively uniform noise that is well correlated with visual impression. The CNR measure used in the paper by Chatuverdi et al. [26] was computed

$$
\mathrm{CNR}=\frac{C}{N}=\sqrt{\frac{2\left(\overline{\hat{s}}_{b}-\overline{\hat{s}}_{t}\right)^{2}}{\left(\operatorname{var}(\hat{s})_{b}+\operatorname{var}(\hat{s})_{t}\right)}}
$$

where $\overline{\hat{s}}_{t}$ and $\overline{\hat{s}}_{b}$ are the spatial average strain for target and background regions respectively and $C$ and $N$ are the contrast and noise defined as

$$
\begin{aligned}
C & =\left(\overline{\hat{s}}_{b}-\overline{\hat{s}}_{t}\right) / \overline{\hat{s}}_{b}, \\
N & =\frac{\sqrt{\left(\operatorname{var}(\hat{s})_{b}+\operatorname{var}(\hat{s})_{t}\right) / 2}}{\overline{\hat{s}}_{b}} .
\end{aligned}
$$

Fig. 4(a) shows the B-scan of the phantom before compression. Fig. 4(b)-(c) shows the elastograms corresponding to an applied compression of $3 \%$ computed from the axial displacement maps estimated with the MRCC and MRCCA methods respectively. Interfaces between regions of large strain contrast decorrelate near the flow channel except within the stiff inclusion where the channel deformation is limited. Computation
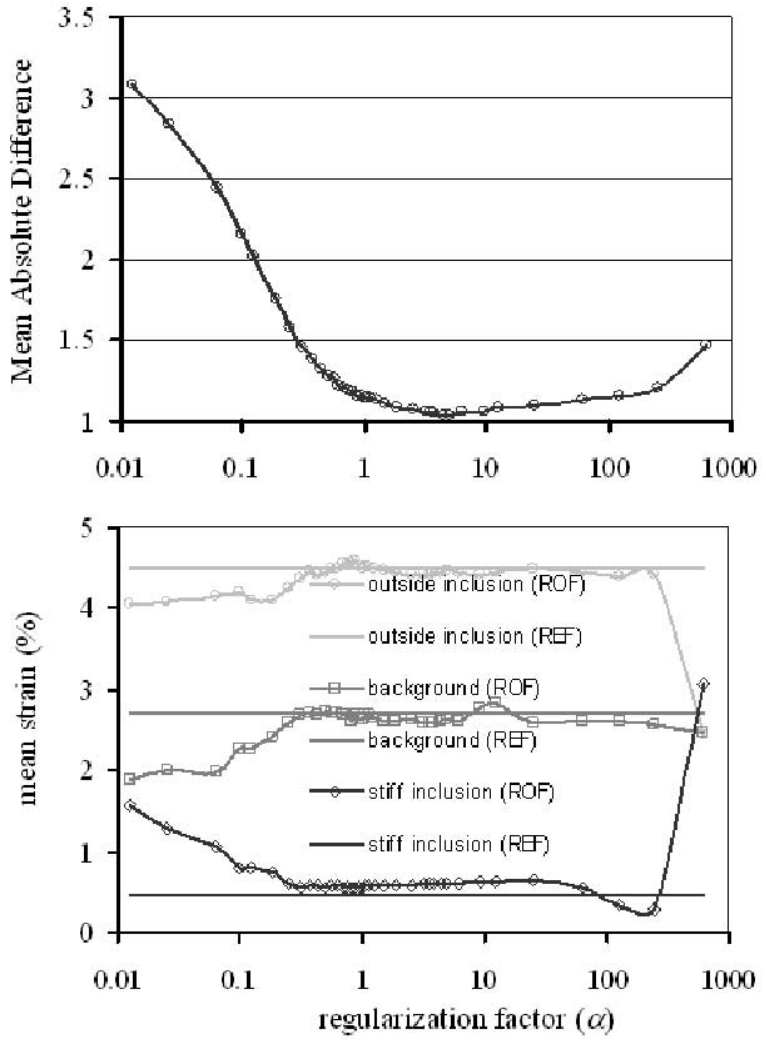

Fig. 5. (a) Mean absolute difference (MAD) between the MRCC elastogram and ROF elastograms reconstructed using different values of the regularization parameter $\alpha$ (abscissa). A stable minimal region is observed for $\alpha=2-10$. (b) Mean strain values as function of $\alpha$ in regions inside the inclusion, near the inclusion and in the background. Corresponding reference mean values obtained with the MRCC method are shown in dotted lines.

times for the MRCC and MRCCA methods were respectively 7.9 and 1.5 min on a Pentium 4-M CPU 1.70-GHz processor. Notice that the adaptive search used in the MRCCA algorithm produces line propagation artifacts in the background due to the continuity constraints [Fig. 4(c)]. We used the MRCC elastogram as a reference to study the influence of the ROF key parameters because it provides unbiased estimates.

The appearance of the ROF elastograms is greatly influenced by the parameter $\alpha$ that balances the relative weight of the smoothness and conservation constraints in the energy equation. A study comparing the elastograms from phantom data obtained using MRCC with that obtained using ROF was performed with various values of $\alpha$. An $\alpha$ value of 4 was found to provide the necessary compromise between conservation of the spatial resolution and noise minimization for the data examined [Fig. 4(d)]. The ROF computation time was $2.2 \mathrm{~min}$. Fig. 4(e) is an image of the difference between the ROF and MRCC elastograms. The channel appears uniformly bright on the ROF elastogram. Major differences between the ROF and MRCC elastograms are concentrated around the channel [Fig. 4(e)]. Regularization has been effective at minimizing decorrelation errors from the complex fluid motion inside the channel. CNR of elastograms were computed using regions of 160 pixels inside [ROI 1 in Fig. 4(b)] and immediately outside the stiff inclusion [ROI 2 in Fig. 4(b)] respectively as the target and background regions. CNR of the MRCC, MRCCA, and ROF methods were respectively 135, 134, and 


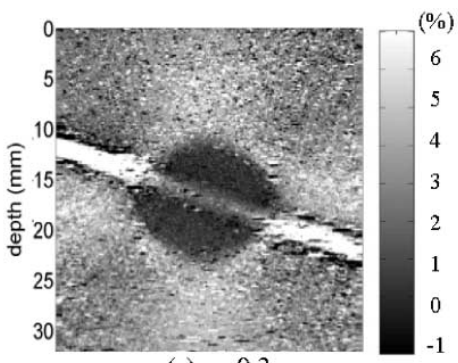

(a) $\alpha=0.3$

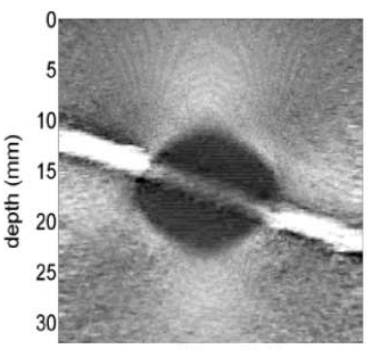

(b) $\alpha=2.5$

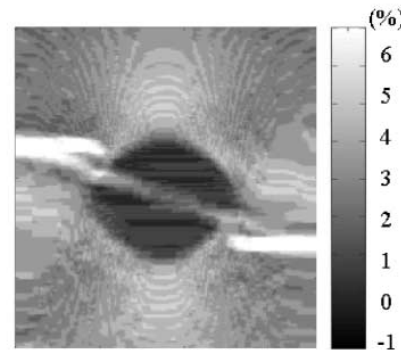

(c) $\alpha=125$
Fig. 6. ROF elastograms of the phantom obtained using (a) $\alpha=0.3$, (b) $\alpha=$ 2.5 , and (c) $\alpha=125$.

145. Regularization reduces target contrast slightly and noise significantly such that there is a net increase in CNR with computation time comparable to the MRCC algorithms.

The mean absolute differences (MAD) between the MRCC elastogram and the ROF elastograms obtained using various smoothing factors $\alpha$ are represented on Fig. 5(a). Mean strain values in small regions inside the inclusion (ROI 1) and immediately outside (ROI 2) as well as in the background (ROI 3) are also represented as function of $\alpha$ on Fig. 5(b). The corresponding mean strain values obtained with the reference MRCC method are shown as straight dotted lines. The MAD curve [Fig. 5(a)] shows that the solution is stable for $2<\alpha<10$. Mean strain values in this range are stable and approximate the reference values [Fig. 5(b)]. The stability of strain estimates is confirmed visually in Fig. 6(b). ROF images are not strongly dependant on $\alpha$ in the range $2<\alpha<10$. For $\alpha$ higher than 10 , the regularization dominates, thus providing a very uniform but inaccurate displacement and strain fields [Fig. 6(c), $\alpha=125$ ]. For $\alpha$ smaller than 2, the regularization is too weak to suppress noise [Fig. 6(a), $\alpha=0.3$ ]. We concluded from these visual and quantitative studies of the influence of the regularization parameter, that values of $\alpha$ that achieve the best compromise between contrast to noise ratio and accuracy of strain estimates must be comprised between 2 and 10 .

The number of spatial scales included in the ROF algorithm was set to $6(I=5)$. Consequently, the initial block size was $128 \times 32$ samples, which is comparable to the block size used in the MRCC method. For $I<5$, both the convergence and optimization time were degraded because the initial size of the data blocks were too large. Values of $I \geq 5$ gave acceptable results, although the computation time increases in proportion to the number of scales for $I \geq 5$.

Parameters that control the extent of the search during displacement estimation, $D_{a}$ and $D_{l}$, were selected adaptively depending on the tissue explored and compression applied. The

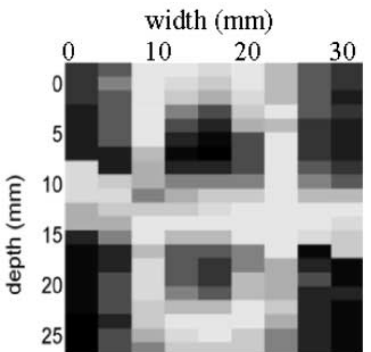

(a) $i=I$

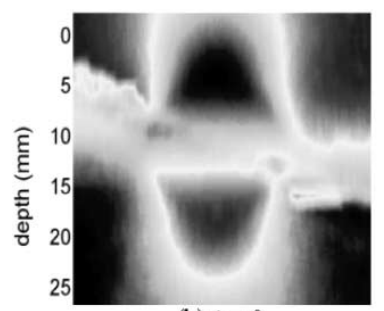

(b) $i=0$

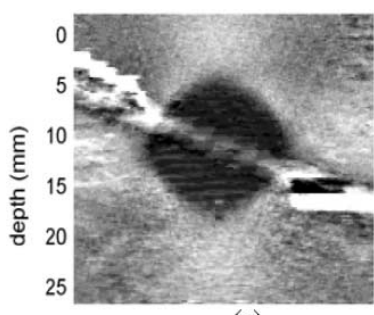

(c)

$\alpha_{I}=\alpha$

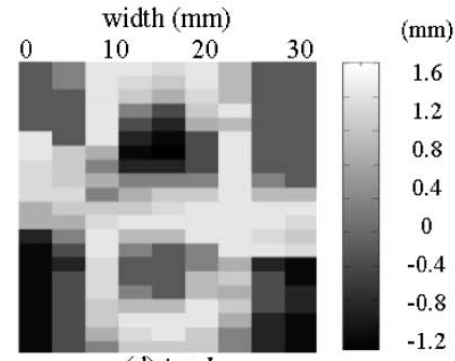

(d) $i=I$

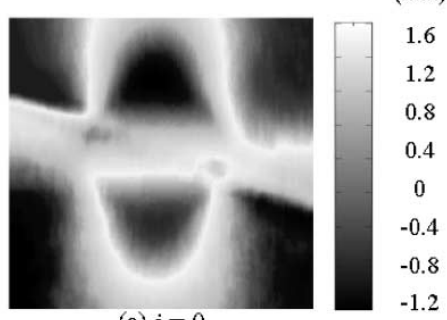

(e) $i=0$

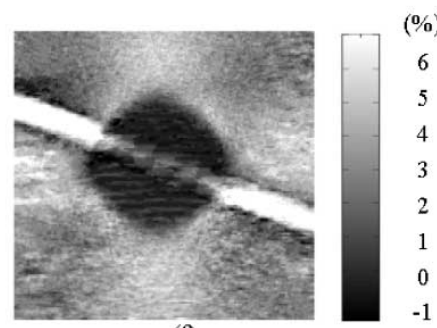

(f)

$\alpha_{I}=0$
Fig. 7. Estimated displacement fields at (a) the coarsest resolution $(i=I)$ using a regularized initialization $\left(\alpha_{I}=\alpha\right)$, (b) the finest resolution $(i=0)$ using $\alpha_{I}=\alpha$, (d) $i=I$ using a nonregularized initialization $\left(\alpha_{I}=0\right)$ and (e) $i=0$ using $\alpha_{I}=0$. Resulting elastograms using (c) $\alpha_{I}=\alpha$ and (f) $\alpha_{I}=0$. Regularization at the coarsest resolution results in a misplacement of the channel (c) while initialization by a nonregularized solution leads to a correct estimate (f).

choice of the search window size is critical since the computation time increases in proportion to each spatial dimension of the window. If the window is too small, the algorithm cannot find the global energy minimum. If the window is too large, time is wasted and there are more opportunities for echo noise to increase estimation uncertainty. The optimal search window size was determined empirically by increasing its dimensions until stable results were found. Compressing the phantom $2 \%$, we found $D_{a}=12$ pixels $(160 \mu \mathrm{m})$ and $D_{l}=4$ pixels $(480 \mu \mathrm{m})$ were optimal.

\section{Influence of the Adaptive Search}

1) Nonregularized Initialization: The classical multiscale approach to optical flow consists of initializing the search with null vector fields and using a regularized model at the coarsest scale $\left(\alpha_{I}=\alpha\right)$. We compared that approach to one modified to use a nonregularized initialization $\left(\alpha_{I}=0\right)$. Fig. 7 shows the resulting displacement maps and elastograms from the phantom data corresponding to $2 \%$ applied compression estimated using $\alpha_{I}=\alpha$ (left column) and $\alpha_{I}=0$ (right column). The post-compressed RF field had been stretched back by $2 \%$ before computing the displacement field in order 
to globally match the precompressed field. This provides a displacement field spatially centered around zero. Estimated displacement fields at $i=5$ (coarsest scale) and $i=0$ (finest scale) are represented on the first two rows. When $\alpha_{I}=\alpha$, uniform blocks are favored from the beginning of the optimization [Fig. 7(a)]. This tends to attenuate abrupt deformation variations near the channel and prevent the solution from evolving toward the global minima [Fig. 7(b)]. The resulting elastogram shows errors in the positioning of the channel [Fig. 7(c)]. When the initial estimation is performed using no regularization $\left(\alpha_{I}=0\right)$, a more realistic displacement map showing abrupt changes is obtained at the top level [Fig. 7(d)] that then evolves toward the global minima [Fig. 7(e)]. The channel is correctly positioned in the resulting elastogram [Fig. 7(f)]. These results were observed whether using the same search window at each scale or using an adaptive window centered on the displacement field found at the previous scale. Thus, the results do not depend on the fact that the search window is adaptive. Although the results are the same, adaptive searches lower the computation times. Optimization using a nonregularized initialization yields $10 \%$ time reduction when compared with that obtained using $\alpha_{I} \neq 0$, due to the fact that only one iteration is required at the top level.

2) Reduced Search Windows: Elastograms from the phantom as well as those from in vivo data were formed using constant and adaptive window sizes. The corresponding images are identical, and Fig. 8 shows that the final energies reached at the end of each scale using constant search windows (gray) and adaptive search windows (stripes) are also identical. Similarly, the number of modified sites and the number of iterations necessary to reach the minimum energy at each scale are the same using the standard and adaptive methods. In the case illustrated here, convergence time is decreased by a factor of two when adaptive windows are used. When comparing the convergence time at each scale, we observe that at levels 5 and 4, the processing times of the two methods are identical because the counting of modified sites begins at level 4 , and consequently the search windows only start to be affected at level 3. Note also that the processing time of the two methods is short at level 5 because only one iteration is necessary. Processing time for the conventional method increases almost quadratically from level 4 to level 0 because the regularity term requires four times more computation every time the resolution increases. However, with the adaptive method, the processing time does not necessarily increase with the level. Here the processing time depends primarily on the number of changes that occurred at the previous scale. The gain in time obtained with the adaptive method is related to the heterogeneity of local deformations and ranges from $30 \%$ to $70 \%$ depending on the investigated object.

\section{Elasticity Imaging of In Vivo Breast Data}

Elastograms of normal breast tissue were formed with the ROF algorithm using the same regularization factor and number of spatial scales found optimal for phantom data ( $\alpha=4$ and $I=$ $5)$. The search window size was adapted to breast tissue properties. For applied compressions $<0.5 \%$, we found that $D_{a}=4$ and $D_{l}=2$ were optimal, and for compressions between $0.5 \%$ and $1.0 \%, D_{a}=8$ and $D_{l}=3$ were used.
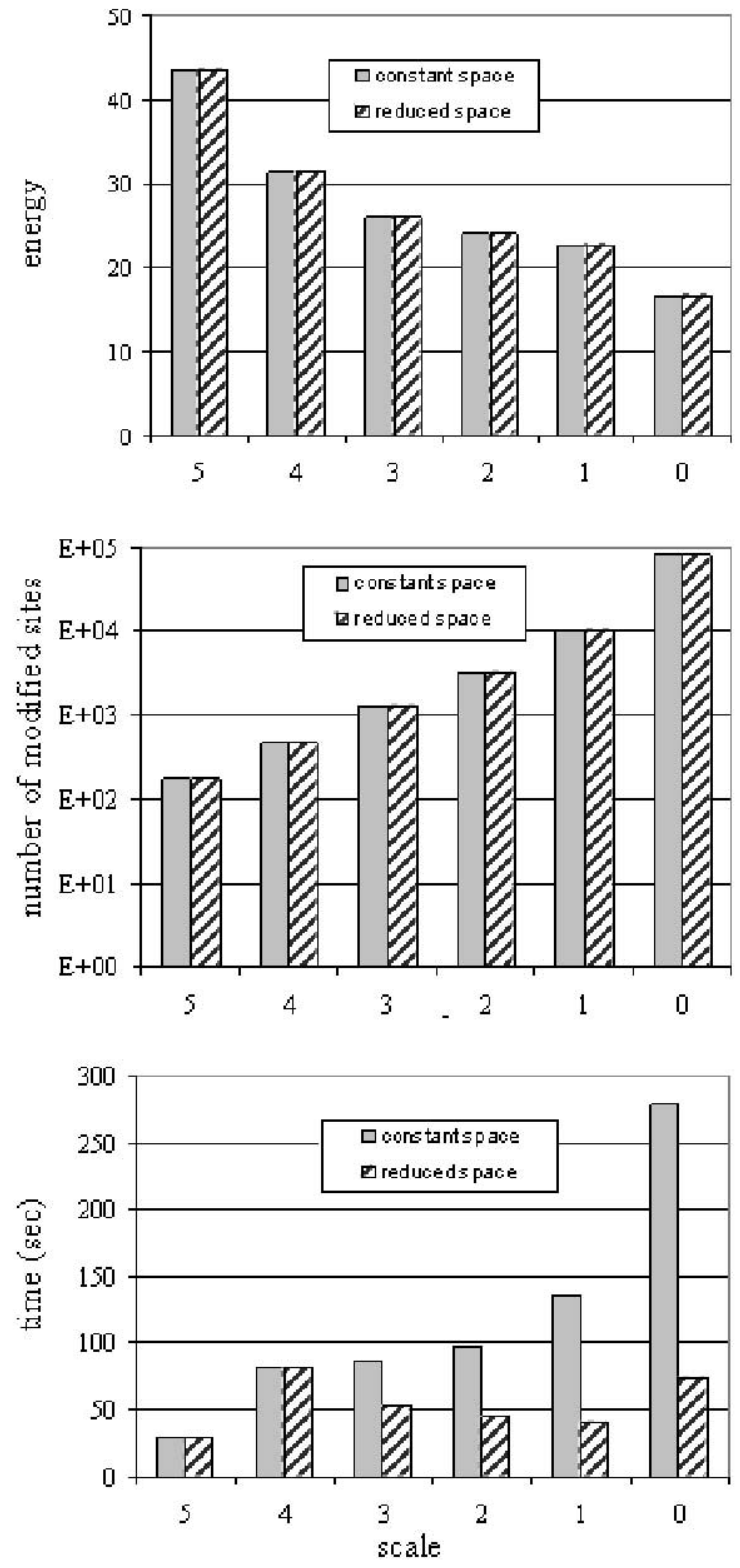

Fig. 8. Comparison as a function of the spatial scale of the energy attained, the number of modified sites and the processing time using constant search windows (gray) and adaptive search windows (stripes). The energies and number of modified sites of the constant and adaptive searches are identical while the total processing time of the adaptive search is half that of the regular search.

Fig. 9(a) shows the B-scan corresponding to normal breast tissue data. On the B-scan, glandular tissue appears hyperechoic while fatty tissue is hypo-echoic. Multicompression acquisition was used in order to obtain high contrast elastograms without high decorrelation noise [39]. Final elastograms were obtained by adding strain estimates from three successive frames each corresponding to a small compression of $0.5 \%$. The elastograms displayed correspond to a $1.5 \%$ net compression. Soft fatty tissue appears bright on the elastograms while the stiffer glandular tissue appears dark. Fig. 9(b)-(c) shows the elastograms obtained respectively using the MRCC and MRCCA methods. The MRCCA method performs slightly better than the MRCC in case of breast tissue, as the MRCCA 


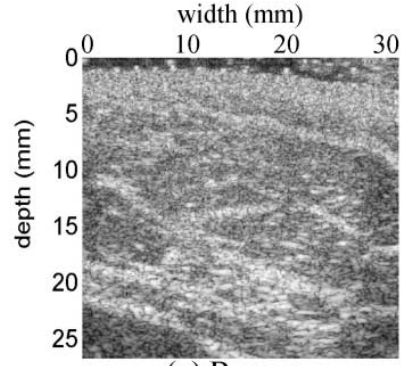

(a) B-scan

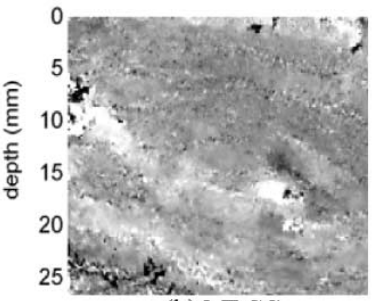

(b) MRCC

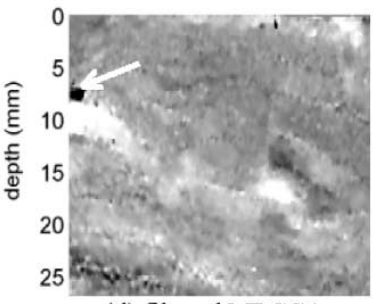

(d) filtered MRCCA

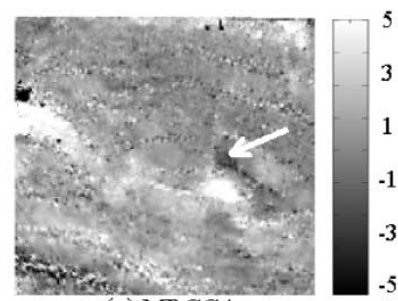

(c) MRCCA

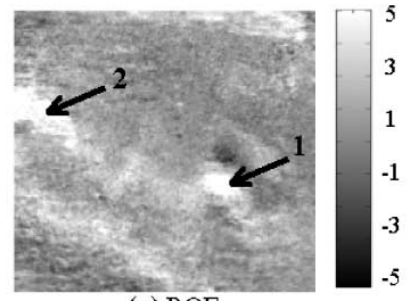

(e) ROF
Fig. 9. (a) B-scan of normal breast tissue. Fat tissue is hypoechogenic and glandular tissue is hyperechogenic. (b)-(c) MRCC and MRCCA elastograms corresponding to $1.5 \%$ compression. Fat tissue appears bright (high strain). A small rigid structure (arrow) is detected. Decorrelation noise is slightly reduced with MRCCA. (d) Median-filtered MRCCA elastogram. The salt-and-pepper effect is smoothed out. (e) ROF elastogram. The decorrelation noise has been eliminated and the small rigid structure still appears clearly.

search constraints help eliminating some decorrelation noise. The elastograms clearly show a $5 \mathrm{~mm}$ dark structure immediately followed by a bright region (arrow). The dark region indicates a stiff structure probably corresponding to an obstructed duct. ROF seems to preserve these important structures while reducing salt-and-pepper decorrelation [Fig. 9(e)]. For comparison purposes, a $3 \times 3$ median filter was applied to the MRCCA elastogram [Fig. 9(d)]. Decorrelation noise is reduced with filtering but the resulting image has less details while noise is reduced without an obvious loss of resolution with the ROF method. Furthermore, large regions of estimated strain errors that are only smoothed with median filtering [such as the dark area indicated by an arrow on Fig. 9(d)], are eliminated with the ROF method.

Table I summarizes the strain contrast $(C)$, noise $(N)$, contrast-to-noise ratio (CNR) and total computation times corresponding to the MRCC, MRCCA, MRCCA filtered and ROF elastograms. Two target regions of 100 pixels corresponding to bright structures were selected together with two adjacent background regions. The target regions are indicated by arrows on Fig. 9(e). The MRCCA filtered and ROF methods seem to present the best performances in terms of CNR and noise. The CNR of the MRCCA filtered method is significantly better than that of all the other methods for the second pair of target and background regions (6.50 compared with $3.67,4.13$, and 4.26 for the MRCC, MRCCA, and ROF methods respectively) while
TABLE I

PERFoRMANCE COMPARISON OF THE MRCC, MRCCA, AND MRCCA FILTERED AND ROF ElASTOGRAMS OF BREAST TISSUE CORRESPONDING to $1.5 \%$ Multicompression. The Mean Strain Values Have BeEN COMPUTED IN ROIs LOCATED IN BRIGHT REGIONS [Fig. 9(e)] AND THEIR ADJACENT BACKGROUND REGIONS

\begin{tabular}{ccccc}
\hline & MRCC & MRCCA & $\begin{array}{c}\text { MRCCA } \\
\text { filtered }\end{array}$ & ROF \\
\hline Mean strain (\%) & & & & \\
target 1 & 6.20 & 6.12 & 6.02 & 6.31 \\
background 1 & 1.19 & 1.20 & 1.19 & 1.19 \\
target 2 & 4.94 & 5.45 & 5.48 & 5.26 \\
$\quad$ background 2 & 1.86 & 2.06 & 2.07 & 2.00 \\
Contrast (C) & & & & \\
t1/b1 & 4.21 & 4.08 & 4.04 & 4.32 \\
t2/b2 & 1.66 & 1.64 & 1.64 & 1.63 \\
Noise (N) & & & & \\
$\quad$ t1/b1 & 0.87 & 0.80 & 0.56 & 0.59 \\
t2/b2 & 0.45 & 0.40 & 0.25 & 0.38 \\
CNR & & & & \\
$\quad$ t1/b1 & 4.84 & 5.08 & 7.18 & 7.35 \\
t2/b2 & 3.67 & 4.13 & 6.50 & 4.26 \\
\hline Total Computation time \\
(min)
\end{tabular}

the ROF method presents the best CNR for the first region pair (7.35 compared with 4.84, 5.08, and 7.18 for the MRCC, MRCCA, and MRCCA filtered methods respectively). Median filtering systematically result in noise reduction with almost no reduction in contrast which leads to this strong improvement of the CNR (about $1.5 \%$ increase). The ROF method also reduces noise compared with the MRCC and MRCCA methods but the reduction is more adaptive. Certain regions are more regularized than others depending on their local characteristics (the noise is reduced by a factor 1.35 in the first ROI pair while it is only reduced by a factor 1.05 in the second). The original MRCC method shows lower performance than the MRCCA and ROF methods both in terms of computation time (18.5 min) and CNR. In fact, the MRCC method seems to not perform as well on in vivo tissue as on phantom data. This can be explained by the fact that biological tissues are composed of many layer interfaces, that result in a loss of signal coherence when subjected to manual uncontrolled deformations. Methods that use a priori information, formulated either implicitly (MRCCA) or explicitly (ROF), are more appropriate to track tissue motion in the presence of decorrelation noise induced by incoherent motion. The MRCCA method is the least computational time extensive method in the example shown $(3.2 \mathrm{~min})$. The ROF method also shows a reasonable computation time (4.9 min). Since the MRCCA and ROF methods use acceleration processes in homogeneous regions, their computation times strongly depend on the explored data. When observing all the cases studied, we can say that computation times of the two methods are overall comparable.

Fig. 10 shows another example of normal breast tissue data. Fig. 10(a) shows the B-mode scan, Fig. 10(b)-(c) shows the elastograms corresponding to about $0.35 \%$ compression obtained using the MRCCA and ROF approaches respectively. Fig. 10(d) shows the MRCCA elastogram obtained from one single compression of about $0.7 \%$. A completely decorrelated elastogram is obtained. Fig. 10(e) shows a multicompression elastogram obtained using the MRCCA method by adding two successive $0.35 \%$ compressions. Decorrelation noise is considerably reduced but is still present. When using the 


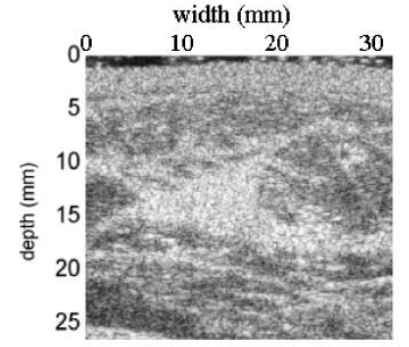

(a) B-Scan

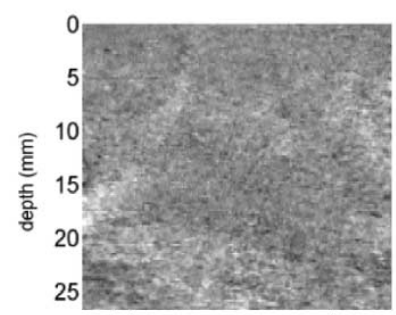

(c) $\varepsilon=0.35 \%-\mathrm{ROF} / \mathrm{SC}$

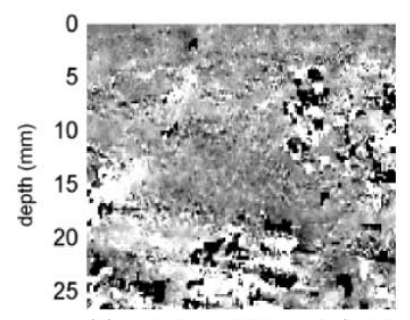

(e) $\varepsilon=0.7 \%-\mathrm{MRCCA} / \mathrm{MC}$

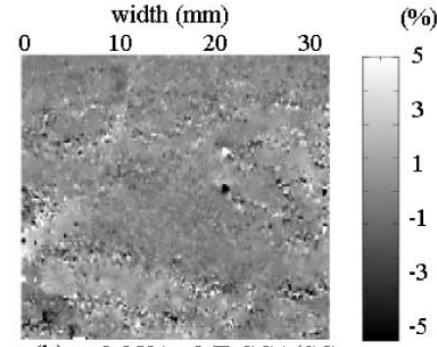

(b) $\varepsilon=0.35 \%-\mathrm{MRCCA} / \mathrm{SC}$

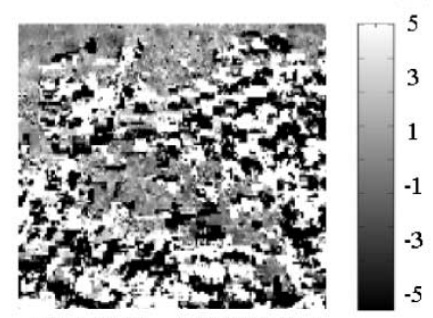

(d) $\varepsilon=0.7 \%-\mathrm{MRCCA} / \mathrm{SC}$

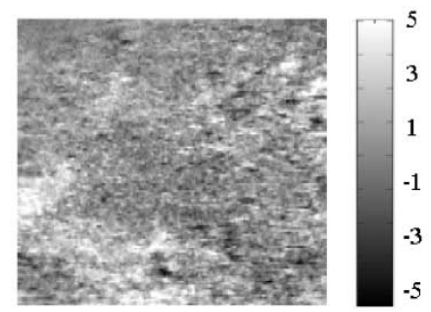

(f) $\varepsilon=0.7 \%-\mathrm{ROF} / \mathrm{SC}$
Fig. 10. (a) B-scan of normal breast tissue. (b) MRCCA elastogram corresponding to $0.35 \%$ compression. (c) ROF elastogram corresponding to $0.35 \%$ compression. (d) MRCCA elastogram corresponding to a single compression of $0.7 \%$. Important decorrelation noise due to out-of-plane motion predominates. (e) MRCCA elastogram corresponding to multicompressions totaling $0.7 \%$. Although strongly reduced, decorrelation noise is still present. (f) ROF elastogram corresponding to a single $0.7 \%$ compression. The regularization permits to lift under-determinations due to incoherent motion and to clear decorrelation noise.

ROF method, elastograms can be reconstructed in a single step at $0.7 \%$ compression [Fig. 10(f)]. The ROF algorithm allows strain computation at larger applied deformations than cross-correlation methods. Regularization is effective at reducing decorrelation noise without distorting the elastogram because tissue deformations are spatially smooth.

\section{SUMmARY AND CONCLUSION}

The paper presents a method for reconstructing elasticity images from ultrasonic RF echo data based on a regularization of the optical flow constraint (ROF). A measure of tissue elasticity is obtained by computing the axial strain derived from local tissue displacements. The displacement field is estimated by minimizing an energy equation that imposes constraints of echo amplitude conservation and displacement smoothness. This approach offers the advantage of combining local and global information, which leads to both accurate and low noise displacement fields. However, the search for the global minimum of energy is very time consuming. In order to accelerate convergence, a coarse-to-fine minimization is used. The algorithm developed is an adaptation of multiscale optical flow methods for ultrasonic elasticity imaging. The method has been generalized to take into account the case of anisotropically sampled data. A major implementation concern was to increase the efficiency of the multiscale optimization process both in terms of convergence and computation time. The general initialization of the optimization as well as the multiscale process were specifically designed for that purpose by using a nonregularized model at the coarsest scale and introducing adaptively reduced configuration spaces.

Optimized parameters of the energy model were determined using a tissue-like phantom and then applied to in vivo data of normal breast tissue. Elastograms obtained with the ROF method were compared with that obtained with a purely multiscale cross-correlation technique (MRCC) and a modified cross-correlation technique that uses some a priori knowledge (MRCCA). Comparative measurements of contrast and noise performances seem to indicate that the regularization improves noise characteristics while preserving contrast. In terms of computation time, the ROF and MRCCA algorithms are comparable. Regularization also seems to visually preserve small structure details, although further thorough validation of the method is necessary to evaluate the performances of the ROF method in terms of resolution. If future analysis reveals that the regularization significantly degrades spatial resolution, a relaxation of the smoothness constraint on contours could be implemented. Clinical interest of the ROF method will also be studied by evaluating its ability to differentiate between benign and malignant breast tumors.

In the present implementation, only one regularization term has been introduced that imposes a $2 \mathrm{D}$ continuity of the displacement field. An additional term of coupling between successive compressions could easily be added to the energy equation to impose a $2 D+t$ continuity of the displacement field. This feature would be particularly appropriate for reconstructing strain sequences to observe, for example, cardiac or vascular deformations. Finally, the present model can be formalized in 3D and the reconstruction problem can be extended to the estimation of $3 \mathrm{D}$ displacement fields.

\section{REFERENCES}

[1] L. Gao, K. J. Parker, R. M. Lerner, and S. F. Levinson, "Imaging of the elastic properties of tissue-A review," Ultrasound Med. Biol., vol. 22, pp. 959-977, 1996.

[2] D. B. Plewes, J. Bishop, A. Samani, and J. Sciarretta, "Visualization and quantification of breast cancer biomechanical properties with magnetic resonance elastography," Phys. Med. Biol., vol. 45, pp. 1591-1610, 2000.

[3] M. M. Doyley, J. C. Bamber, F. Fuechsel, and N. L. Bush, "A freehand elastographic imaging approach for clinical breast imaging: System development and performance evaluation," Ultrasound Med. Biol., vol. 27, pp. 1347-1357, 2001.

[4] K. M. Hiltawsky, M. Kruger, C. Starke, L. Heuser, H. Ermert, and A. Jensen, "Freehand ultrasound elastography of breast lesions: Clinical results," Ultrasound Med. Biol., vol. 27, pp. 1461-1469, 2001.

[5] A. Manduca, T. E. Oliphant, M. A. Dresner, J. L. Mahowald, S. A. Kruse, E. Amromin, J. P. Felmlee, J. F. Greenleaf, and R. L. Ehman, "Magnetic resonance elastography: Non-invasive mapping of tissue elasticity," Med. Image Anal., vol. 5, pp. 237-254, 2001.

[6] S. Y. Emelianov, X. Chen, M. O'Donnell, B. Knipp, D. Myers, T. W. Wakefield, and J. M. Rubin, "Triplex ultrasound: Elasticity imaging to age deep venous thrombosis," Ultrasound Med. Biol., vol. 28, pp. 757-767, 2002.

[7] C. L. de Korte and A. F. W. van der Steen, "Intravascular ultrasound elastography: An overview," Ultrasonics, vol. 40, pp. 859-865, 2002. 
[8] K. J. Parker, L. Gao, R. M. Lerner, and S. F. Levinson, "Techniques for elastic imaging: A review," IEEE Eng. Med. Biol. Mag., vol. 15, pp. 52-59, Nov.-Dec. 1996

[9] L. S. Wilson, D. B. Robinson, and M. J. Dadd, "Elastography-The movement begins," Phys. Med. Biol., vol. 45, pp. 1409-1421, 2000.

[10] J. Ophir, B. Garra, F. Kallel, E. Konofagou, T. Krouskop, R. Righetti, and T. Varghese, "Elastographic imaging," Ultrasound Med. Biol., vol. 26, pp. 23-29, 2000.

[11] I. Hein and W. O'Brien, "Current time-domain methods for assessing tissue motion by analysis from reflected ultrasound echoes-A review," IEEE Trans. Ultrason. Ferroelect. Freq. Contr., vol. 40, pp. 84-102, Mar. 1993.

[12] M. F. Insana, L. T. Cook, and P. Chaturvedi, "Analytical study of bioelasticity ultrasound systems," in Proc. 16th Int. Conf Information Processing in Medical Imaging (IPMI'99), 1999, pp. 1-14.

[13] F. Kallel and M. Bertrand, "Tissue elasticity reconstruction using linear perturbation method," IEEE Trans. Med. Imag., vol. 1, pp. 299-313, June 1996.

[14] L. Soualimi, M. Bertrand, R. Mongrain, and J. Tardif, "Forward and inverse problems in endovascular elastography," Acoust. Imag., vol. 23, pp. 203-209, 1997.

[15] M. M. Doyley, P. M. Meaney, and J. C. Bamber, "Evaluation of an iterative reconstruction method for quantitative elastography," Phys. Med. Biol., vol. 45, pp. 1521-1540, 2000.

[16] L. Han, A. Noble, and M. Burcher, "The elastic reconstruction of soft tissues," in Proc. IEEE/EMB Int. Symp. Biomed. Imag., 2002, pp. $1035-1038$.

[17] E. E. Konofagou, T. Varghese, and J. Ophir, "Spectral estimators in elastography," Ultrasonics, vol. 38, pp. 412-416, 2000

[18] J. Ophir, I. Cespedes, H. Ponnekanti, Y. Yazdi, and X. Li, "Elastography: A quantitative method for imaging the elasticity of biological tissues," Ultrason. Imag., vol. 13, pp. 111-134, 1991.

[19] J. Bai, C. Ding, and Y. Fan, "A multi-scale algorithm for ultrasonic strain reconstruction under moderate compression," Ultrasonics, vol. 37, pp. $511-519,1999$

[20] C. Ding and J. Bai, "Peak position estimation algorithms for cross-correlation function in elastography," in Proc. 20th Annu. Int. Conf. IEEE Eng. Med. Biol. Soc., vol. 20, 1998, pp. 866-868.

[21] F. Yeung, S. F. Levinson, and K. J. Parker, "Multilevel and motion model-based ultrasonic speckle tracking algorithms," Ultrasound Med. Biol., vol. 24, pp. 427-441, 1998.

[22] T. Varghese, M. Bilgen, and J. Ophir, "Multiresolution imaging in elastography," IEEE Trans. Ultrason. Ferroelect. Freq. Contr., vol. 45, pp. 65-75, 1998.

[23] E. Brusseau, C. Perrey, P. Delachartre, M. Vogt, D. Vray, and H. Ermert, "Axial strain imaging using a local estimation of the scaling factor from RF ultrasound signals," Ultrason. Imag., vol. 22, pp. 95-107, 2000.
[24] J. Dey, J. J. Mai, and M. F. Insana, "Multiresolution approach to strain imaging," in Proc. IEEE Ultrasonics Symp., vol. 2, 2000, pp. $1853-1856$

[25] P. Chaturvedi, M. F. Insana, and T. J. Hall, "2-D companding for noise reduction in strain imaging," IEEE Trans. Ultrason. Ferroelect. Freq. Contr., vol. 45, pp. 179-191, Jan. 1998.

[26] - "Testing the limitations of 2-D companding for strain imaging using phantoms," IEEE Trans. Ultrason. Ferroelect. Freq. Contr., vol. 45, pp. 1022-1031, July 1998.

[27] C. Pellot-Barakat, J. J. Mai, C. Kargel, A. Herment, B. Trummer, and M F. Insana, "Accelerating ultrasonic strain reconstructions by introducin mechanical constraints," Proc. SPIE, vol. 4684, pp. 323-333, 2002.

[28] B. Horn and B. Schunck, "Determining optical flow," Artif. Intell., vol. 17, pp. 185-203, 1981.

[29] G. Mailloux, A. Bleau, M. Bertrand, and R. Petitclerc, "Computer analysis of heart motion from two-dimensional echocardiograms," IEEE Trans. Biomed. Eng., vol. BME-34, pp. 356-364, 1987.

[30] A. Giachetti, "Matching techniques to compute image motion," Image Vis. Comp., vol. 18, pp. 247-260, 2000.

[31] M. F. Insana, T. J. Hall, P. Chaturvedi, and C. Kargel, "Ultrasonic properties of random media under uniaxial loading," J. Acoust. Soc. Amer. vol. 110, pp. 3243-3251, 2001.

[32] P. Perez and F. Heitz, "A multiscale approach to image analysis using Markov random fields," Trait. Signal, vol. 9, pp. 459-472, 1992.

[33] R. Laganière and A. Mitiche, "Direct bayesian interpretation of visual motion," Robot. Auton. Syst., vol. 14, pp. 247-254, 1995.

[34] F. Heitz, P. Perez, and P. Bouthemy, "Multiscale minimization of global energy functions in some visual recovery problems," CVGIP; Image Understanding, vol. 59, pp. 125-134, 1994.

[35] L. N. Bohs and G. E. Trahey, "A novel method for angle independent ultrasonic imaging of blood flow and tissue motion," IEEE Trans. Biomed. Eng., vol. 38, pp. 280-286, Mar. 1991.

[36] S. Geman and D. Geman, "Stochastic relaxation, Gibbs distribution and the bayesian restoration of image sequences," IEEE Trans. Pattern Anal. Machine Intell., vol. PAMI-6, pp. 721-741, 1984

[37] J. Besag, "On the statistical analyis of dirty pictures," J. Roy. Statist. Soc. $B$, vol. 48, pp. 259-302, 1986.

[38] J. J. Mai and M. F. Insana, "Strain imaging of internal deformation," Ultrasound Med. Biol., vol. 28, pp. 1475-1484, 2002.

[39] T. Varghese, J. Ophir, and I. Cespedes, "Noise reduction in elastograms using temporal stretching with multicompression averaging," Ultrasound Med. Biol., vol. 22, pp. 1043-1052, 1996. 